\title{
Pig in a china shop: a personal perspective of how pandemic (H1N1) 2009 affected the work of intensive care units in NSW
}

\author{
Richard J. Totaro \\ Intensive Care Service, Royal Prince Alfred Hospital \\ Email: rtotaro@mail.usyd.edu.au
}

\begin{abstract}
Intensive care support of patients with severe respiratory failure has improved over recent years and with improved methods of ventilation fewer of these patients now die. However despite these improvements, a significant number of patients still die with acute respiratory failure. The outbreak of pandemic (H1N1) 2009 influenza presented NSW intensive care units with many critically ill patients with severe acute respiratory failure. Three case studies and summary data illustrate the impact of these patients on intensive care units services in Australia and New Zealand.
\end{abstract}

The day-to-day of a busy intensive care unit (ICU) is complex and challenging; new admissions, long-stay patients and bureaucratic considerations all demand attention. The mind is focused, however, when faced with something new.

Pandemic (H1N1) 2009 influenza was something new. Almost over one weekend in June $2009,{ }^{1}$ ICUs in New South Wales (NSW) were faced with a set of patients suffering a form of acute severe hypoxemic respiratory failure that appeared significantly worse than in previous flu seasons. In addition unlike other flu seasons, 'a third of our patients were young or middle-aged adults who neither were pregnant nor had a known coexisting condition'. ${ }^{1}$

The severity of the respiratory failure resulted in the author's hospital (Royal Prince Alfred Hospital [RPAH]), a tertiary referral hospital in NSW, receiving referrals for extra corporeal membrane oxygenation (ECMO). ECMO is a method of providing oxygen and removing carbon dioxide in patients whose lungs are so damaged that standard mechanical ventilation is insufficient. It requires the insertion of large intravenous catheters, and the circulation of the patient's blood through a pump to an artificial lung. With the development of new equipment, ECMO (which was previously only offered by large tertiary hospitals) can now be initiated by specialist teams in smaller facilities prior to transferring the patient to a tertiary centre for ongoing management. With this improved method of ventilation, fewer of these patients now die. ${ }^{2}$ In NSW, RPAH, St Vincent's Hospital and the NSW Ambulance Service, with funding from the NSW Government, have developed an ECMO Retrieval Service that manages these patient transfers. ${ }^{3}$

Over the next 2 months this increase in the number of patients with severe respiratory failure continued. Two recent publications describe the experience of ICUs in Australia and New Zealand in responding to the pandemic. . $^{1,3}$

From June to August 2009, ICUs in Australia and New Zealand ventilated 722 patients with confirmed H1N1 influenza infection. Of these, 103 died (14.3\%). Nine percent of these patients were pregnant or in the postpartum period (compared with approximately $1 \%$ of the general population of Australia and New Zealand). ${ }^{1}$ A total of $28.6 \%$ of patients had a body mass index (BMI) over 35 (compared with approximately $5.3 \%$ of the Australian adult population in 2003). ${ }^{1}$ The peak percentage of all ICU beds occupied by H1N1 influenza patients varied by region across Australia and New Zealand from 9 to $19 \%$.

Of the over 200 ICUs in Australia and New Zealand, only 15 provide ECMO. These units admitted 252 (H1N1) 2009 influenza patients, ventilated 201 and undertook ECMO in 68. Of this sickest group of patients who received ECMO, $14 \operatorname{died}(21 \%) .{ }^{1,4}$

The effect on the work of the ICU posed by this surge of patients with acute respiratory failure was dramatic and tested resources. At RPAH, the Intensive Care Service managed 31 patients with confirmed pandemic (H1N1) 2009 influenza. Of these, 19 required ECMO. 


\section{Case studies}

Following are three case studies of patients seen by the RPAH Intensive Care Service that illustrate the severity of the illness, the diagnostic problems encountered when dealing with novel diseases and some of the impacts on health workers of this outbreak of influenza.

\section{Case 1}

Typical of the patients RPAH treated was the third referral for ECMO, a 37-year-old woman. Her family had been sick with the flu the week before she presented to her local hospital, short of breath. Her breathing became progressively worse, with low oxygen (hypoxia) and high carbon dioxide (hypercapnia) levels in the blood. She required intubation into her lungs and mechanical ventilation. Over the next hours her oxygen levels continued to deteriorate despite progressive increases in the amount of mechanical ventilation she received, reaching a point where it was likely that the ventilation could potentially damage her lungs.

Consequently she was referred to RPAH for consideration for ECMO. The ECMO team was mobilised late on a Saturday afternoon to the referring hospital. The team placed the vascular access lines and treatment commenced, with a dramatic improvement in her condition within minutes. Due to restrictions in transport times, the team had to spend the night at the referring hospital before she could be transported to Sydney in a specially-equipped ambulance.

She also required the insertion of a tracheostomy to facilitate her mechanical ventilation.

While she was on ECMO her lungs became essentially unventilatable, with the size of each breath less than the volume used for a baby.

The course of this patient's illness was also remarkable for the length of time it took for her lungs to begin to recover. After 3 weeks on ECMO her lungs had recovered enough to allow the removal of ECMO. It took another 2 weeks for her to be separated from the ventilator. She was discharged from the ICU to a ward, then to a rehabilitation centre prior to returning home. At a recent follow-up, apart from some hair loss, she is very well and is active.

\section{Case 2}

A 20-year-old man was referred, his likely contact being a friend who was suffering from the flu. He was previously well apart from having a BMI of more than 30 . He was referred to RPAH from interstate and was initiated on ECMO before being transferred. Initial pernasal swabs taken at his referring hospital and at RPAH were negative for H1N1 influenza. A careful repeat sample using bronchoalveolar lavage collection from his lungs confirmed
(H1N1) 2009 infection and that the initial sampling to be a false negative finding. This was a problem frequently encountered during this outbreak of pandemic (H1N1) 2009 influenza, and may reflect either migration of the viral replication into the lung or poor pernasal sampling techniques. It is not explained well as yet. This patient required ECMO for over a week, but was eventually able to be separated from the extracorporeal support and the ventilator. He was returned to his referring hospital where he was eventually discharged well.

\section{Case 3}

A male doctor - who had worked the previous week despite having flu-like symptoms - was admitted to the ICU with marked hypoxia. It was assumed that he had pandemic (H1N1) 2009 influenza at his work. He was previously very fit and recovered following a few days of high dependency support including high flow oxygen therapy. His physical debility however caused concern among the staff who cared for him who identified with a fellow healthcare worker. Among other things his experience reinforced the need for the mandatory personal protective regimen which was highly adhered to. The RPAH Intensive Care Service is not aware of any staff member who developed pandemic (H1N1) 2009 influenza from work related exposure.

\section{Conclusion}

In Australia, pandemic (H1N1) 2009 influenza placed a significant burden on ICU services, and at its peak accounted for almost $25 \%$ of ICU beds. The patients who were most affected by this new illness were younger than the usual ICU patients, pregnant or immediately post-partum, or likely to have a high BMI. However, ICU services were able to manage the work load presented and to provide highly resource-intensive interventions with both excellent survival rates and excellent health outcomes for these patients.

\section{References}

1. The ANZIC Influenza Investigators, Webb SA, Pettila V, Seppelt I, Bellomo R, Bailey M, Cooper DJ et al. Critical Care Services and 2009 H1N1 Influenza in Australia and New Zealand. N Engl J Med 2009; 361(20): 1925-34. doi:10.1056/ NEJMoa0908481

2. Erickson SE, Martin GS, Davis JL, Matthay MA, Eisner MD and the NIH NHLBI ARDS Network. Recent trends in acute lung injury mortality: 1996-2005. Crit Care Med 2009; 37(5): 1574-9. doi:10.1097/CCM.0b013e31819fefdf

3. NSW Department of Health. New medical retrieval service for critically ill patients. Available from: http://www.health.nsw. gov.au/news/2009/20090721_00.html (Cited 2009-12-23.)

4. The Australia and New Zealand Extracorporeal Membrane Oxygenation (ANZ ECMO) Influenza Investigators. Extracorporeal Membrane Oxygenation for 2009 Influenza A (H1N1) Acute Respiratory Distress Syndrome. JAMA 2009; 302(17): 1888-95. doi:10.1001/jama.2009.1535 